4 | 2017

Technologies numériques et diffusion de l'information pendant les périodes de conflits et de crise dans le monde

\title{
Questions épistémologiques à propos d'une « interdiscipline »
}

La communication internationale dans l'univers global des sciences sociales

Epistemological questions about an "interdiscipline". International communication in the global universe of social sciences

Questões epistemológicas sobre a "interdisciplinar". A comunicação

internacional no universo total de ciências sociais

Ndiaga Loum

\section{OpenEdition}

1 Journals

Édition électronique

URL : http://journals.openedition.org/ctd/835

DOI : $10.4000 /$ ctd.835

ISSN : 2491-1437

Éditeur

Chaire Unesco Pratiques émergentes en technologies et communication pour le développement

Référence électronique

Ndiaga Loum, «Questions épistémologiques à propos d'une « interdiscipline » », Communication, technologies et développement [En ligne], 4 | 2017, mis en ligne le 04 septembre 2017, consulté le 29 mars 2021. URL : http://journals.openedition.org/ctd/835 ; DOI : https://doi.org/10.4000/ctd.835

Ce document a été généré automatiquement le 29 mars 2021.

Communication, technologies et développement 


\title{
Questions épistémologiques à propos d'une « interdiscipline »
}

\author{
La communication internationale dans l'univers global des sciences
} sociales

Epistemological questions about an "interdiscipline". International communication in the global universe of social sciences

Questões epistemológicas sobre a "interdisciplinar". A comunicação

internacional no universo total de ciências sociais

Ndiaga Loum

\section{Introduction}

1 Peut-on dater la naissance de la communication internationale dans l'univers global des sciences sociales? Situer les débuts de la communication internationale n'est pas tâche aisée. Chaque auteur, selon l'aspect qu'il voudrait privilégier dans son analyse, fixera un point de départ spécifique et différent. D'ailleurs, la difficulté probable à s'entendre sur la question expliquerait que très peu s'aventuraient à affirmer de manière péremptoire: "Ici commence la communication internationale». Cette prudence axiologique ne nous est pas étrangère. Dès lors, ce que nous faisons dans cette présente réflexion, ce ne serait pas de tenter une petite histoire de la communication internationale. C'est plutôt, à la façon d'Armand Mattelart, une sorte d'interprétation, d'analyse des grands évènements qui ont marqué l'évolution des communications internationales et conduit petit à petit cette matière, dans l'univers général de la communication, vers une "distanciation en voie d'autonomisation ». Ce processus devant normalement mener vers une autonomie, pour l'instant, impossible. Ce qui confinerait à lui attribuer de façon provisoire le statut d' «interdiscipline » ou même d' «indiscipline». Tant, ceux qui ont compétence et légitimité à s'exprimer au nom de la «communication internationale» appartiennent à divers univers disciplinaires. 
2 Étant entendu que la rigueur et l'objectivité seront d'autant plus facilement obtenues que l'on aura visé un objectif plus réduit (Grawitz, 1993), nous ne pouvions ici déroger à une entreprise de délimitation. Ce qui suppose des éléments qu'on privilégie, certains qu'on néglige, d'autres qu'on laisserait carrément en rade. Autrement dit, un parti pris scientifique s'impose. Cela dit, il convient d'admettre que les réseaux de communication façonnent considérablement l'organisation de la planète : "L'interconnexion généralisée des économies et des sociétés est, en effet, le point d'aboutissement du mouvement vers l'intégration mondiale qui a débuté au tournant du XIXe siècle. Élargissant progressivement la sphère de circulation des personnes ainsi que des biens matériels et symboliques, les dispositifs de communication ont hâté l'incorporation des sociétés particulières dans des ensembles de plus en plus vastes, et n'ont eu de cesse de déplacer les frontières physiques, intellectuelles et mentales " (Mattelart : 1996, p. 3). Pour dessiner cette topographie des réseaux de communication à l'échelle internationale, les stratégies des acteurs divergent même si l'objectif commun est d'établir un échange " transfrontières » facilité par les progrès techniques. Si les uns évoquent la sécurité mutuelle et l'interdépendance des nations, d'aucuns véhiculent des messages marqués par l'œcuménisme religieux, d'autres mettent en branle les intérêts économiques avec le «pragmatisme de l'entreprise et l'impératif catégorique de la division internationale du travail» (Mattelart, 1996, p.3). Enfin, certains avancent des arguments teintés d'universalisme et investissent les avancées technologiques d'une capacité à réaliser le rêve de la démocratie planétaire où les « exclus » retrouveraient leur droit de participer, de dire et d'échanger. Si l'histoire de la communication internationale, cette "mal nommée " dont parlait Mattelart est toujours le parent pauvre de la recherche sur la communication, elle risque malheureusement de ne pas retrouver ses «lettres de noblesses» dans cette présente analyse. Cette tâche nécessaire et pourtant urgente serait immense. Elle nécessiterait plus de temps et de moyens et explorerait des chemins si larges qu'ils ne pourraient être ouverts que par les chantiers d'une thèse. Ce que nous ne faisons pas ici. Notre ambition si " générale » soit-elle, est plus modeste, elle ne prétend pas à l'exhaustivité, même si elle embrasse plusieurs domaines, lesquels nous paraissent essentiels dans la dynamique de la construction d'une réflexion qui interroge de façon critique et tente de fixer les grandes lignes de cette « interdiscipline » ou cette « indiscipline » qu'est la communication internationale. Une autre remarque préalable s'impose : c'est que, si l'on ne peut pas s'empêcher de répertorier et d'analyser les «moments forts" de l'évolution de la communication internationale, il n'empêche que notre rejet de tout déterminisme technologique apparaisse à la lecture de cet article. Notre conviction étant qu'aucune analyse des médias n'est crédible si elle ne tient pas compte du contexte historique, économique, politique, social. Autant alors dire tout de suite que nous nous démarquons du "médiacentrisme " ignorant complètement le contexte social, culturel et historique. Les rapports à la communication ne sont qu'un moment et un lieu des rapports sociaux qui les déterminent. Autant donc affirmer avec force notre distance par rapport à tout déterminisme technique et toutes les techno-utopies qui prétendent rendre compte de l'histoire des étapes par lesquelles est passée l'humanité. Cela explique que nous ne cédons pas à la facilité qui aurait fait qu'on reprenne une à une les différentes innovations technologiques dans le domaine des communications au cours de l'histoire, pour en faire le corolaire d'une évolution linéaire de la communication internationale. Une telle démarche, si elle était la nôtre, nous 
amènerait à négliger les contextes culturel, politique économique, bref à renoncer à une critique sociale des usages de ces techniques.

3 Ces précisions préalables étant faites, on peut à présent énoncer la structure qui va abriter le cheminement de notre pensée sur cette question. Dans un premier temps nous aborderons le contexte d'émergence de la communication internationale, étant entendu bien sûr qu'on parte du postulat que celle-ci reste inséparable de la modernité. Dans un deuxième temps, nous interrogerons la pertinence d'une problématique qui associe la communication internationale et la dynamique de la mondialisation, phénomène dont on ne saurait la séparer, autant dans son expression du projet capitaliste à vocation globalisante que dans les rejets qu'elle semble susciter (antimondialisation et altermondialisation). Enfin, nous clorons cette réflexion sur l'identification et l'analyse de défis variables et valsant au gré de la géopolitique internationale. Ces questions que l'on peut situer dans une réflexion globale sur la communication démontrent tout autant la difficulté à lui tracer des frontières qui la sépareraient des autres disciplines des sciences sociales, comme les relations internationales et la science politique, par exemple.

\section{La communication internationale : produit ou exigence de la modernité}

Il va falloir d'abord dire quelques mots sur ce qui alors distingue la société moderne de la société traditionnelle ? $^{1}$ C'est Ferdinand Tönnies (1887) qui au XIXe siècle consacre leurs premières désignations en donnant à la société traditionnelle le nom de Gemeinschaft et à la société moderne celui de Gesellschaft ${ }^{2}$. Tönnies a cherché à établir clairement la distinction entre la communauté traditionnelle (la tribu, le clan, la famille paysanne élargie, la corporation artisanale...) au sein de laquelle l'individu était écrasé par le poids de la collectivité et celui des traditions qu'il respectait, et la société moderne, celle qui, partant d'un individu supposé libre et rationnel, va déléguer une partie de ses pouvoirs aux institutions dites modernes et à l'État, l'autre partie étant librement exercée dans la sphère de la vie privée. Il n'existe aucun doute qu'à l'époque de Tönnies, le passage de la communauté traditionnelle à la société moderne, entamé dès la fin du XVIIe siècle, représentait intellectuellement et de manière pratique, un énorme progrès.

Paul Attallah (1989) fournit dans une remarquable synthèse les éléments de comparaison susceptibles de traduire les changements intervenus lors du passage de la société traditionnelle à la société moderne. Cette dernière se caractérise ainsi, à la différence de la société traditionnelle, par l'urbanisation, l'industrialisation, la massification, la spécialisation, le contrat. On retiendra ici l'idée d'un changement social observable dans le temps et de façon continue. Ce changement finit par transformer la structure et le fonctionnement de l'organisation sociale d'une collectivité et modifier ainsi le cours de son histoire. Les éléments susceptibles de changements sont par exemple, la création de centres urbains, la structure de la population active, les règles de la vie sociale (familles, entreprise, écoles, etc.), le contrôle social.

6 Ces changements ne sont pas sans conséquence sur les comportements de la société : développement de l'individualisme, modification des liens sociaux, affaiblissement 
progressif et déclin des hiérarchies anciennement établies. Ces changements sociaux sont très importants et sont à lier avec le changement profond des structures économiques. Le changement social est ainsi inséparable du développement économique. Cela, parce que le développement économique s'accompagne d'une modification de la nature de la production, du cadre de vie, du travail (séparation du lieu de travail et du lieu de vie de la famille), de la structure sociale et des formes d'identification collective (classe ouvrière). D'où la récurrente question sur l'origine des changements sociaux. Le changement social est-il uniquement lié à des aspects économiques? D'autres facteurs influent- ils sur le changement social? Là intervient une distinction entre les facteurs exogènes (progrès techniques ou économiques, causes démographiques, émergence de valeurs nouvelles comme l'individualisme) et les facteurs endogènes (rôle des conflits sociaux, approfondissement d'une valeur existante comme la tendance à l'égalité) dans l'explication du changement social.

7 Pour ce qui concerne les facteurs exogènes, on se souviendra de l'importance donnée par Durkheim (cité par Aron, 1967) ${ }^{3}$ aux conséquences de la croissance démographique. Il voyait dans cette croissance démographique un facteur de diversification et d'intensification des rapports sociaux qui finissent par créer de l'interdépendance et de la complémentarité. Pour sa part, Max Weber insiste sur l'apparition de nouvelles valeurs induites par le protestantisme: la réussite matérielle incite les individus à rechercher la perfection dans les activités économiques (d'où l'épargne, l'investissement et la croissance économique) (Weber, cité par Aron, 1967). Weber situe ainsi les changements sociaux dans les transformations de rationalités liées à des phénomènes souterrains (développement progressif de l'organisation étatique avec le phénomène de bureaucratisation $)^{4}$.

8 Concernant les facteurs endogènes, Karl Marx (cité par Aron, 1967) ${ }^{5}$ va mettre en évidence l'importance des rapports sociaux, des conflits de classes pour expliquer les changements dans la société, par exemple le passage du féodalisme au capitalisme. Toujours, à propos de ces facteurs endogènes, Alexis de Tocqueville (cité par Aron, 1967) souligne une tendance à l'égalité présente dans toute société démocratique et qui peut se traduire par des changements conséquents et divers, mais pas forcément positifs : recherche du bien-être, individualisme, indépendance, risque de perte de la libertét.

9 C'est là également que réside l'importance de la contribution historique d'Immanuel Wallerstein (1990) 7 qui figurerait en bonne place dans bon nombre de syllabus des cours de communication internationale. Devenu une référence incontournable, à notre humble avis, l'article de Wallerstein, connu sous l'appellation du "système-monde " mérite ici une étude particulière. Son originalité est de complexifier la problématique tout en s'inscrivant dans la dynamique des théories visant à expliquer jusque-là le passage de la société traditionnelle à la société moderne.

Nous avons synthétisé supra les grandes théories de la transformation sociale (celles de Durkheim, de Tönnies, de Marx et de Weber). Wallerstein estime qu'avant lui, on ne s'est jamais posé la question de savoir si le développement du capitalisme était inévitable. Tout au plus, on se limitait à expliquer pourquoi le capitalisme, la modernité, le développement industriel et la croissance intensive avaient d'abord eu lieu en Occident. La problématique de Wallerstein cherche à inverser cette tendance; elle consiste à se demander pourquoi ces évènements se sont produits quelque part, car 
toutes les explications fournies jusque-là évoquent des variables qui avaient existé en des endroits bien différents.

11 Si nous évoquions ces auteurs et le processus historique des transformations sociales qu'ils cherchent à décrire, c'est surtout pour marquer la nécessité de situer une réflexion sur la communication internationale dans l'analyse de l'univers global des sciences sociales. L'évocation de travaux comme ceux de Wallerstein nous rappelle l'obligation épistémique de problématiser la question globale de la communication internationale en lien avec la dynamique de la mondialisation et du projet « universel » du capitalisme dont elle serait le moteur.

Ce qu'il convient enfin de retenir à propos du passage de la société traditionnelle à la société moderne, c'est que plus les rapports sociaux se complexifient, plus les relations économiques se délocalisent, plus se pose la question de la facilitation des échanges. Et c'est là qu'intervient la dimension fonctionnelle de la communication internationale. Alors, si l'économie n'est pas le seul facteur explicatif du changement social, elle est un des éléments déterminants.

13 Dès lors, le développement économique, avec les transformations sociales induites par la modernité, accompagne la communication dont les progrès sont tout aussi inséparables de ce contexte. Et Armand Mattelart $(1996$, p. 6) ne s'y trompe pas dès lors qu'il affirme de façon catégorique que :

"L'invention de la communication comme idéal s'est faite sous le signe des idées de modernité et de perfectibilité des sociétés humaines». L'auteur souligne que la "libre communication de la pensée et des opinions" nargue les frontières et rappelle les propos de Diderot au siècle des Lumières, écrits dans sa Lettre sur le commerce de la librairie rédigée vers 1763: «Bordez, Monsieur, toutes vos frontières de soldats, armez-les de baïonnettes pour repousser tous les livres dangereux qui se présenteront, et ces livres, pardonnez-moi l'expression, passeront entre leurs jambes et sauteront par-dessus leurs têtes et nous parviendront. » (cité par Armand Mattelart, 1996, p. 6).

Ces réflexions préliminaires qui situent la communication internationale dans la dynamique «englobante» des changements, de l'évolution historique et des transformations sociales, ne dispensent pas d'un besoin de définition. Il va falloir alors dire qu'est-ce que l'on entend par communication internationale?

\section{De la nécessité de définir la communication internationale et de la problématiser}

D'abord, comment définir la communication? Les définitions de la communication sont nombreuses et plusieurs auteurs s'y sont essayés. Mais, pour saisir la dimension internationale de celle-ci, la définition de Wolton (1997) nous paraît être la plus complète. Elle mérite donc ici d'être reprise. Pour Wolton, la communication est d'abord un idéal d'expression et d'échange à l'origine de la culture occidentale (Wolton, 1997), elle présuppose l'existence d'individus libres et égaux. On suppose toutes les batailles menées depuis le XVIIe siècle pour promouvoir ces concepts qui sont inséparables de la modernité (Wallerstein, 1993). Elle serait ensuite, toujours selon Wolton, l'ensemble des médias de masse, de la presse à la radio, et à la télévision qui, en un siècle, ont bouleversé les rapports entre la communication et la société. Puis, l'auteur affirme que la communication renvoie à l'ensemble des nouvelles techniques 
de communication, qui, à partir de l'informatique, des télécommunications, de l'audiovisuel et de leur interconnexion, ont, en moins d'un siècle, contribué à modifier les conditions d'échange, mais aussi de pouvoir au niveau mondial. En dehors de sa dimension instrumentale et technique, la communication aurait donc une dimension symbolique importante. La communication serait ainsi, résume Wolton (1997), un ensemble de valeurs, symboles et représentations qui organisent le fonctionnement de l'espace public des démocraties de masse, et plus généralement, de la communauté internationale à travers l'information, les médias, les sondages, l'argumentation et la rhétorique.

16 Si l'information a pour objet de mettre en forme le monde, de rendre compte des évènements, elle est inséparable de la communication, qui, au-delà de l'idéal d'échange et d'interaction, constitue le moyen de diffuser ces informations et de construire les représentations. Les deux sont inséparables selon Dominique Wolton (1997).

17 Par communication donc, il faut entendre l'ensemble des techniques, de la télévision aux nouveaux médias, et leur implication économique, sociale et culturelle, mais aussi les valeurs culturelles, les représentations et les symboles liés au fonctionnement de la société moderne et de la démocratie (Wolton, 1997).

Pourquoi lierait-on le qualificatif "internationale» au terme communication? La raison paraît évidente, mais elle mérite quelques clarifications et surtout une problématisation. L'évidence, c'est que la communication ne saurait être cantonnée à un pays ou une région donnés qui alors en auraient l'exclusivité. Les nécessités liées à l'existence des sociétés humaines ont entraîné des dynamiques (migrations, échanges, conflits, déplacements, etc.) qui durant toute l'histoire, ont justifié et accompagné l'invention de moyens de communication. Il peut donc paraître comme un euphémisme de parler de communication internationale, celle-ci étant même un aspect des relations internationales.

19 Seulement, il y a lieu de problématiser pour ne pas tomber dans la simplification à l'extrême. Ceci explique que nous cherchions à articuler la communication internationale au contexte de mondialisation marqué par les avancées technologiques, les phénomènes de convergence et de concentration, la crise de l'État-nation, la création des multinationales, la vocation globalisante du capitalisme, les résistances altermondialistes, les nouvelles formes de gouvernance, etc. Cette problématisation justifie alors qu'on s'intéresse aussi à la définition de la mondialisation.

\section{Définir la mondialisation}

Que devrait-on entendre par mondialisation? Marc Raboy voit la mondialisation comme un «contexte général caractérisé par le rôle diminué des États nationaux, la concentration transnationale du pouvoir économique capitaliste, la réduction des contraintes de temps et d'espace, la remise en question des notions d'identités nationale et culturelle, l'émergence des nouveaux réseaux globaux et la mise en place progressive d'un nouveau régime juridicopolitique de gouvernance mondiale » (Raboy, 1999, p. 58). Cette définition, sans être exhaustive, a le mérite de souligner les multiples aspects du terme qui renvoie non seulement à la logique de l'expansion du capitalisme et la capacité qu'ont les tenants de cette même logique à imposer des contraintes légales, règlementaires et politiques au monde entier. Dans cette perspective, tous les biens sont marchands, y compris les biens culturels et symboliques, y compris donc 
aussi, la communication. Voilà pourquoi une problématique de la communication internationale associée à la mondialisation, ne peut exclure la volonté voilée ou non du projet capitaliste de s'étendre dans tous les pays du monde, d'abord considérés comme des marchés ouverts.

Cependant, il serait naïf de penser que cette volonté d'imposer un modèle unique fondé sur le laisser-faire et le laisser-aller propres à la logique capitaliste et libérale se fait sans résistance. D'où la pertinence de s'intéresser aussi aux mouvements dits altermondialisation ou antimondialisation, dès lors qu'on aborde la problématique de la mondialisation. Ce que d'ailleurs, les tenants de la mondialisation capitaliste et les mouvements altermondialistes ont en commun, c'est l'usage des moyens de communication, mais pour des raisons différentes, contradictoires pour ne pas dire diamétralement opposées.

La mondialisation serait donc avant tout un phénomène économique et politique, mais aussi un phénomène social et culturel. La mondialisation renvoie également à une nouvelle ère de communications, un idéal de partage de l'information, une possible uniformisation des cultures. Les avancées technologiques dans le domaine des communications et leurs capacités à atteindre la plus grande masse avaient ainsi mené à la célèbre formule de Marshal Mc Luhan : «village global » $(1967)^{8}$ ? La sémantique traduisant une telle réalité glissera par la suite, et cela grâce aux rapides évolutions technologiques, vers ce que l'on nommera la "société de l'information ». La réalité de cette société de l'information devant aussi être questionnée dans une perspective résolument critique (George et Granjon, 2008), même si l'on ne peut nier qu'elle reste un projet non détachable d'une réflexion globale sur le capitalisme et la mondialisation, deux phénomènes qui consacrent la diminution du rôle de régulation politique et règlementaire des États (Vitalis, 1999) ${ }^{9}$. La mondialisation comme phénomène à la fois politique et économique a aussi un lien historique avec les grands conflits mondiaux ayant marqué le XXe siècle.

\section{Les conflits mondiaux : vers un monde de plus en plus divisé}

La première moitié du XXe siècle est marquée par deux guerres mondiales qui entraînent des alliés et des colonies dans des conflits. Les grands empires coloniaux vont payer un lourd tribut dans ces confrontations qui contribuent à affaiblir considérablement leurs économies. Les empires britanniques et français se voient donc progressivement supplantés par les États-Unis qui vont sortir définitivement de leur « isolationnisme » lors de la 2e guerre mondiale.

Si les deux guerres mondiales (1914-1918 et 1939-1945) stoppent la dynamique de la mondialisation sous la poussée de la logique capitaliste, on peut aussi y lire l'arrivée sur la scène mondiale d'une nouvelle puissance, qui va au contraire, contribuer à l'accélérer, mais pour ses intérêts exclusifs ou tout moins principaux: les États-Unis (Post, 2011). L'affaiblissement des puissances coloniales européennes à cause des efforts de guerre va favoriser l'émergence d'une nouvelle force dominante qui a eu tout le temps de se construire pendant que les autres cherchaient à se neutraliser (Post, 2011).

Le discours de Truman sur l'État de l'Union, le 20 janvier 1949, indiquait clairement au reste $d u$ monde et surtout aux pays du tiers-monde la voie à suivre, celle du développement «chevillé » à la conception américaine du capitalisme : "Il nous faut lancer un nouveau programme qui soit audacieux et qui mette les avantages de notre 
avance scientifique et notre pensée industrielle au service de l'amélioration et de la croissance des régions sous-développées. Plus de la moitié des gens de ce monde vivent dans des conditions voisines de la misère... Les États-Unis occupent parmi les nations une place prépondérante quant au développement des techniques industrielles et scientifiques $»^{10}$. Le plan Marshall ${ }^{11}$ proposé aux pays européens pour les aider à reconstruire leurs économies en ruine en est la meilleure preuve. Cette période va ainsi préparer un nouvel "âge d'or" du capitalisme dans sa version américaine. Les indicateurs marquants de cette période sont la forte production de biens de consommation de masse et la croissance économique accélérée grâce à la reconstruction de l'Europe avec le Plan Marshall. On assiste à une production à grande échelle et à coûts décroissants des produits standardisés parmi les biens de consommation durables (auto, électroménager, télévision, etc.). Parallèlement à cette production, se développent progressivement des classes moyennes qui achètent des biens de consommation. On constatera aussi le rôle croissant de l'État qui prend en charge des activités de plus en plus nombreuses (agriculture, santé, éducation, etc.).

Toutefois, si le capitalisme se développe et que les échanges se multiplient à l'échelle internationale, il est à signaler que les années 60 sont marquées par des contestations sociales importantes : mouvement anticapitaliste, mouvement féministe, mouvement pacifiste et mouvement de décolonisation. Le monde se divise en trois blocs avec en plus des blocs capitaliste et communiste, les pays dits «non-alignés ».

L'arrivée de ce mouvement des pays dits non-alignés sur la scène internationale est capitale dans le domaine des communications internationales. Ils transportent le débat sur le déséquilibre de l'information au sein de l'UNESCO dans les années 70 et 80, avec notamment la création de la commission Mac Bride en 1977 qui va tenter d'offrir des solutions pour rétablir un équilibre entre pays du Nord et pays du Sud dans les flux communicationnels en général et les communications internationales, en particulier ${ }^{12} \boldsymbol{2}$. La fin des années 60 est marquée par la saturation des marchés et par la baisse des profits qui remet en cause les fondements mêmes du système capitaliste (Boltanski et Chiapello, 1999). Plusieurs solutions sont trouvées pour augmenter la croissance : les consommateurs doivent remplacer leurs produits le plus vite possible (publicité et innovation); de nouveaux marchés doivent être ouverts à des entreprises privées et dans les services; de nouveaux marchés doivent émerger dans les pays où il y a des consommateurs potentiels.

En somme, la libre circulation des marchandises devient un impératif de compétitivité et permet aux entreprises de bénéficier de coûts du travail moins élevés. C'est dans cette dynamique que s'enclenchent les initiatives de délocalisation des entreprises pour aller là où la production est moins coûteuse, avec une main-d'œuvre disponible et bon marché. Mondialisation et marchandisation vont donc de pair. On parie sur le fait que le commerce international amène la paix entre les peuples. Le rapport de forces reste très défavorable aux travailleurs, et la richesse créée est de plus en plus concentrée dans le cadre du développement du capitalisme financier profitant à une petite minorité d'investisseurs ${ }^{13}$. Parallèlement, les États semblent voir leur rôle diminuer au profit de structures internationales comme l'Organisation mondiale du commerce (OMC) qu'ils ont mises en place pour réguler paradoxalement un monde d'échanges fondé sur la motivation expresse de la dérégulation et de la dérèglementation.

Si ces quelques lignes démontrent le lien de causalité entre le capitalisme et la mondialisation, il va falloir s'interroger sur le rôle des communications pour substituer 
au duo capitalisme-mondialisation le trio capitalisme-mondialisation-communication ? Va-t-on vers une mondialisation "humanisée» par la dimension solidaire de la communication normative (Wolton, 1997 et 2003)? Ou bien, va-t-on vers l'instrumentalisation de la communication (et surtout des nouvelles technologies de l'information et de la communication) pour servir d'adjuvant à la mondialisation, le tout au service du projet unificateur du capitalisme ? L'éthique de la discussion et de la communication d'Habermas (1992) est-elle universalisable dans un contexte interculturel marqué aussi par le déséquilibre des échanges culturels, les fractures sociales et économiques qui expliquent toutes les autres fractures qui suivent, et notamment celle dite "numérique»? Bref, quel sens donner à la société dite de l'information et de la communication? La création et la diffusion des technologies de l'information suffisent-elles à conclure à la réalisation de l'idéal d'échange à la source des premières définitions de la communication $($ communicare $=$ partager $)$ et de l'information (informare = échanger) ? Quel sens donner à la notion de "village global » élaborée par Marshall Mc Luhan et rêvée par Wiener qui voyait dans la communication une sorte de "thérapeutique " universalisable pour corriger les anomies sociales et mettre fin aux conflits ? ${ }^{14}$ Cette utopie est-elle réalisable à l'heure de la société dite de l'information marquée par le développement exponentiel des technologies de l'information et de la communication? La fin des utopies politiques (effondrement du communisme à la suite de la chute du mur de Berlin en 1989) laissera-t-elle place à l'avènement d'une nouvelle utopie fondée sur la communication?

«Grâce aux autoroutes de l'information, ces réseaux modernes qui vont transformer la planète, tous les hommes deviennent frères. Telle est la nouvelle utopie - la nouvelle idéologie - qui se répand en cette fin de siècle » ironisait Armand Mattelart dans un article du Monde Diplomatique intitulé «Dangereux effets de la globalisation des réseaux ", en août 1995. Selon Mattelart, l'effondrement récent des grandes utopies politiques a conduit certains penseurs à proposer la communication comme un modèle de remplacement, seule capable de créer entre les hommes ce lien qui fonde les communautés et permet la cohésion sociale. Il s'agirait même d'un antidote contre les dangers de la désorganisation et du chaos, du sous-développement dans les pays du Sud: "A cet égard, ajoutait-il, les nouvelles technologies excitaient tout particulièrement les imaginations; beaucoup voyaient dans le multimédia et les réseaux interactifs de type Internet, les bases d'une cyber-société plus conviviale, plus solidaire et plus démocratique. Les classes sociales seraient effacées, les affrontements disparaîtraient $»^{15}$.

\section{Vers une autre mondialisation?}

Plusieurs questions nous interpellent: le creusement des inégalités économiques tendrait-il à favoriser les migrations internationales? Assiste-t-on en plus de la mondialisation économique à une mondialisation plus politique depuis la chute du mur de Berlin ? On a pu espérer qu'avec la chute du mur de Berlin, on pourrait assister à un renouveau du multilatéralisme autour de l'ONU. Des initiatives comme la conférence des Nations Unies pour l'environnement et le développement à Rio en 1992 ont pu renforcer cette intuition. Mais, les États-Unis prônent un «nouvel ordre mondial » qui s'avère impérial. Cette volonté de domination planétaire des États-Unis est même théorisée par certains auteurs ${ }^{16}$. 

" une américanisation du monde ${ }^{17}$ ", c'est-à-dire une extension à l'échelle de la planète du « rêve américain ». Tandis que Pierre Hassner (cité par Guéhenno, 1999), après avoir passé en revue les différents ouvrages américains qui, depuis dix ans, ont fourni les références intellectuelles du débat international, affirme que, si les États-Unis peinent à transformer le monde selon leurs souhaits, ils sont sans rival aucun pour l'interpréter. Hassner, comme Guehenno, font le constat d'une domination intellectuelle du débat international par les Américains qui serait, selon eux, la touche finale d'une domination à la fois militaire, économique, technologique, médiatique, culturelle et sociétale. Après le Pentagone, Wall Street, CNN, Hollywood, Mc Donald, il y a Microsoft, symbole de la mondialisation électronique à l'Américaine.

Ces idées renvoient incontestablement à celle déjà fortement suggérée par le politologue et conseiller à la Maison-Blanche Joseph Nye (1990), à travers le concept de "soft power", décrit comme "la capacité à atteindre les résultats désirés dans les affaires internationales par l'attraction plutôt que par la contrainte en persuadant les autres de suivre, ou les amener à accepter les normes et les institutions qui produisent le comportement désiré ${ }^{18}$. Le soft power reposerait ainsi sur «l'attrait (appeal) qu'exercent les idées, la capacité à fixer l'agenda de façon à modeler les préférences des autres ». Si, concluait l'auteur, un État réussit ainsi à faire légitimer son pouvoir aux yeux des autres et à instaurer les institutions internationales qui les encouragent à endiguer ou limiter leurs activités, il n'a plus guère besoin de dépenser autant ses ressources économiques et militaires traditionnellement coûteuses. Il apparaît d'ailleurs, si l'on en croit Guéhenno (1999, op. cit.), que cet attrait ait déjà fait ses effets, ce qui explique, aux yeux de cet auteur, pourquoi les citoyens américains sont de moins en moins intéressés à voir leur gouvernement se mêler de ce qui se passe à l'extérieur ; un extérieur qu'ils considèreraient comme une copie, une imitation de ce qui se passe aux États-Unis. Autrement dit, tant que le reste du monde mange les produits de McDonnald, boit du Coca-Cola, achète les logiciels de Microsoft et le dernier Ipad de Apple, et est branché sur Facebook ou les autres réseaux sociaux populaires, il faut s'en féliciter et ne point le considérer autrement, ou s'en préoccuper plus que de besoin.

Si tout cela est avéré, on comprend alors pourquoi l'ancien ministre des Affaires étrangères de la France, Hubert Védrine, disait que le qualificatif «superpuissant » ne suffirait plus pour qualifier les États-Unis et qu'il faille donc plutôt parler d' «hyperpuissance » (Védrine cité par Guéhenno, 1999). Mieux encore, on comprend pourquoi quand le reste du monde s'inquiète des effets de la mondialisation, les ÉtatsUnis s'en réjouissent, parce que celle-ci reste un phénomène dont ils contrôlent les soubassements: "Les régulations globales qui rendent la mondialisation possible continuent d'être assurées pour une part essentielle par des centres de décision situés en Amérique...Il n'y a donc pas aux États-Unis le sentiment de dépossession qui peut exister dans des pays subissant des normes élaborées sans leur participation ». (Guéhenno, 1999, p. 10)

Une réflexion sur la communication internationale est donc inséparable d'une analyse des stratégies de propension du capitalisme et de la mondialisation, "version américaine", dans tous les cas. Les technologies de l'information et de la communication sont-elles alors au service de cette stratégie de séduction, de persuasion du modèle capitaliste américain dont parle Joseph Nye, cité plus haut? La réponse est positive selon Boltanski et Chiapello: «Pour maintenir son pouvoir de 
persuasion, le capitalisme va puiser des ressources en dehors de lui-même, dans les croyances qui possèdent à un moment du temps, un pouvoir important de persuasion » (Boltanski et Chiapello, 1999, p. 59).

Ce qu'il faut comprendre ici, c'est que la communication internationale est aussi un enjeu des rapports des forces. Le processus de création, de diffusion des techniques des communications ne peut être neutre. Ce processus obéit aux intérêts des industries qui $\mathrm{y}$ trouvent une heureuse coïncidence entre le potentiel de création des technologies à engendrer des bénéfices et le sentiment d'utilité pour les usagers qui s'en servent. C'est la rencontre de l'intérêt et de l'utilitarisme qui, selon Bosc (2011), est une sorte de « technologie du social qui vise à construire une subjectivité compatible avec le marché capitaliste $»^{19}$. Le désir d'émancipation et de transparence fort des usagers qui s'exprime à travers ces technologies de la communication, notamment les nouveaux médias dits sociaux, empêche de voir a priori l'intérêt capitaliste à l'origine de leur création et qui est aussi, in fine, le moteur de leur sophistication. Comme le dit Libaert (2003, p. 21), « nous vivons sur l'idée qu'il convient de communiquer plus et mieux pour être heureux et que la modalité effective permettant la communication réside dans l'accroissement de la transparence ".

L'entremêlement entre la logique capitaliste et la communication paraît alors évident aux yeux de plusieurs auteurs, c'est ce que confirme ces propos de Bouquillion et Matthews (2009) : «Les discours sur la société de l'information ou sur la société de la connaissance décrivent l'alliance des technologies de l'information et de connaissance, du marché et de l'individu comme étant au cœur des nouvelles sociétés ${ }^{20}$.

Bien avant même les « aveux » de Joseph Nye reproduits supra, Herbert Shiller (1976, p. 9) attirait l'attention sur les « dangers » de "l'impérialisme culturel » qu'il définissait comme "l'ensemble des processus par lesquels une société est introduite au sein du système moderne mondial et la manière dont sa couche dirigeante est amenée, par la fascination, la pression, la force ou la corruption, à modeler les institutions sociales pour qu'elles correspondent aux valeurs et aux structures du centre dominant du système ou à s'en faire le promoteur $»^{21}$. L'on constatera simplement ici que si Nye et Schiller arrivent à une même définition de l'impérialisme culturel, leurs objectifs scientifiques n'étaient pas les mêmes. Tout au contraire. Quand le premier s'en félicite pour témoigner de la mission humaniste et « globalisante » des États-Unis, le deuxième déplore et dénonce une telle intention aux soubassements idéologiques dévastateurs pour le reste du monde réduit au statut de consommateur des produits culturels américains. La communication interculturelle comme théorie intégrée dans la discipline ou la sous-discipline qu'est la communication internationale trouve ici un terrain fertile pour alimenter le débat scientifique ${ }^{22}$. Le débat sur les menaces de l'impérialisme culturel et la nécessaire défense des identités culturelles, réactualisé par la signature et la ratification de la Convention internationale sur la diversité culturelle de l'UNESCO (2005), implique un renouvellement de la problématique qui associe culture, communication et développement ${ }^{23}$.

\section{Les années 2000 : résistance culturelle, psychose terroriste et nouvelle crise du capitalisme}

Si nous restons dans cette problématique qui associe dans une réflexion critique les notions de communication internationale, de mondialisation et de capitalisme, force 
est de constater que le marché est resté insensible aux critiques considérées trop à gauche des mouvements sociaux. Le mouvement altermondialiste manifeste de la résistance et continue de s'exprimer, mais ses actions sont quelquefois violemment réprimées (sommets du G8 au Canada en 2002 et en Allemagne en 2007). Il est aussi traversé par une profonde crise sur les stratégies et les actions à mener. La seule chose sur laquelle l'ensemble des mouvements altermondialistes semble être d'accord est leur rejet du capitalisme et de son moteur la mondialisation, ce qui se traduit par une dénonciation vigoureuse de leurs effets sur les masses laborieuses et pauvres. Mais l'alternative commune au système capitaliste reste un vœu pieux tant les trajectoires historiques de ces divers mouvements semblent parallèles, voire parfois complètement contradictoires. Ne serait-il pas d'ailleurs plus pertinent de garder la qualification «anti » plutôt «alter» face au phénomène de la mondialisation pour décrire rigoureusement l'action de ces mouvements?

Du côté politique, les Nations-Unies sont affaiblies par l'approche de plus en plus unilatérale des États-Unis qui, au nom de la guerre contre le terrorisme, développe une stratégie à géométrie variable : action collective lors de la première guerre en Irak (1991) et en Afghanistan, et action isolée lors de la deuxième intervention en Irak (2003). Les évènements du 11 septembre 2001 entraînent les États-Unis dans une sorte d'obsession sécuritaire. De nouvelles législations, au diapason de la menace terroriste, ciblent directement le contrôle de l'information et particulièrement l'usage des nouvelles technologies de l'information et de la communication ${ }^{24}$. La communication participe ainsi de l'internationalisation de la lutte contre ce que Bush nomme «l'Axe du mal ». La communication sera mise au service de la guerre contre le terrorisme.

\section{Vers la cyberguerre états-unienne}

Dans leur croisade antiterroriste, les USA se préparent même à la cyberguerre. Ils avaient lancé le plus vaste réseau d'espionnage des télécommunications (surnommé «Echelon ») au temps de la guerre froide, ils ont annoncé qu'ils lanceraient un plan visant à leur assurer le leadership dans le cyberespace. Cette initiative s'inspire du projet Manhattan, qui avait permis de réaliser la première bombe atomique, et se fixe plusieurs objectifs : surveiller le trafic Internet mondial ainsi que les requêtes des moteurs de recherche; créer des chevaux de Troie informatiques pour prendre le contrôle de n'importe quel ordinateur; créer un simulateur d'Internet (et de ses utilisateurs) afin de tester les scénarios d'attaque et de défense et d'entraîner les cyberunités militaires.

Mais, c'est sans compter avec Wikileaks qui fonde sa motivation sur la réappropriation de la communication par les plus faibles contre les puissants? Est-il donc étonnant que le fondateur du site d'informations Wikileaks, Julian Assange soit accusé par les ÉtatsUnis de complot et de complicité avec les auteurs des actes de terrorisme en acceptant de publier des informations considérées comme des actes confidentiels essentiels pour la sécurité nationale américaine et la sécurité à l'échelle mondiale ? Est-ce le moindre des paradoxes que le citoyen américain Edward Snowden, ancien employé de la CIA, soit contraint à l'exil en Russie après avoir livré au site Wikileaks des informations sur les programmes massifs de surveillances des autorités américaines? Cette problématique qui pose la question d'une opposition de valeurs entre les défenseurs à tous crins de la liberté d'expression et ceux qui veulent instaurer une société de 
contrôle, de la surveillance (Vitalis 1981 et 2016 ; Mattelart, 2007 ; Mattelart et Vitalis, $2014)^{25}$ a une forte pertinence conjoncturelle (Loum et Corriveau 2014) tant les cas qui illustrent ces contradictions que charrie la société dite de l'information sont nombreux.

\section{À l'heure du bilan : des défis variables et valsant au gré de la géopolitique internationale}

43 Si le reste du monde occidental compatit avec les États-Unis profondément touchés par les évènements du 11 septembre 2001, il ne partage pas la façon dont les USA mèneront la riposte. Cela se traduit par un désordre dans le camp occidental qui fait face à l'une plus des grandes menaces pour sa sécurité face à la guerre asymétrique menée par les réseaux terroristes (Al Qaeda, puis L'État islamique et ses L'Europe semble réussir son Union économique, mais peine à parler de la même voix sur le plan politique. Ce qui consacre l'affaiblissement de son influence dans le champ des relations internationales. Un affaiblissement accentué par le retrait récent de la Grande-Bretagne de l'Union européenne après un référendum populaire (juin 2016). La Corée du Nord et l'Iran défient le camp occidental en continuant leur programme nucléaire. Même si pour ce dernier pays, l'accord historique récemment conclu avec l'Agence mondiale de l'énergie atomique (2015) a débouché sur la levée de l'embargo et ouvert un nouveau chantier de coopération favorisé par l'arrivée du «camp » des modérés au pouvoir iranien. La Russie, nostalgique du passé, se repositionne et manifeste ses désirs de puissance en attaquant d'abord son voisin, la Géorgie, en encourageant ensuite la dislocation de l'Ukraine, en menant enfin des manœuvres solitaires en Syrie dans le but de consolider le pouvoir de Hassad rejeté par les puissances occidentales. La Chine avance à "visage fermé » avec l'ambition de dominer le monde, mais le fait, contrairement aux Américains, "sans tambour ni trompette». L'expansion économique de la Chine est contrebalancée par la volonté manifeste du régime communiste de contrôler l'Internet et de limiter la liberté de la presse.

Le monde arabo-musulman reste divisé sur sa position face aux stratégies américaines : les complices ou amis (Arabie Saoudite, Koweït, etc.), les ennemis (Syrie), les modérés (Égypte, Algérie, Tunisie, etc.). Mais la révolte sociale en Tunisie et la chute du régime de Ben Ali sur fond de revendication pour une grande liberté d'expression et de démocratisation des médias, rappelle le mot de De Gaule : «les États n'ont pas d'amis, ils ont des intérêts ». Les États occidentaux qui avaient soutenu les dirigeants de ce pays longtemps considéré comme un modèle de stabilité et de relative réussite économique, furent les premiers à se désolidariser d'eux lorsque le peuple tunisien, en quête de liberté, est sorti violemment dans la rue pour chasser le «clan Ben Ali » du pouvoir. La révolution du Jasmin a certes fait des émules, mais, dans bien des cas (Libye, Égypte, Syrie), on frôle le chaos? Si la communication se libère dans le monde arabe, ce sera finalement au bénéfice de qui ? ${ }^{26}$ L'Afrique Noire, elle, ne compte pas sur la table des grandes décisions, mais est conçue comme un nouveau marché avec ses terres vierges offertes aux nouveaux développeurs: les compagnies multinationales de télécommunications y réalisent des bénéfices énormes, ce qui pose la question de la souveraineté économique des États africains incapables de gérer en toute indépendance un secteur qui génère autant d'économies. L'Afrique subit les menaces terroristes dans sa partie ouest (Nigéria, Niger et Mali) affectée par les dégâts collatéraux du chaos libyen consécutif à la chute de Khadafi (ancien président de la Libye) orchestrée par les 
États occidentaux. Avant de penser à tirer profit des nouvelles opportunités qu'offrent les communications internationales en termes économiques (la part importante de l'économie de service dans le domaine des télécommunications), la préoccupation est d'abord sécuritaire dans un contexte d'États-Nations hyper fragile. Les promesses d'un développement accéléré par l'appropriation des technologies de l'information et de la communication (Lerner, Rodgers) ${ }^{27}$, si elles sont d'actualité, doivent nécessairement être envisagées dans une perspective critique comme nous le rappelions supra ${ }^{28}$. Sur le plan économique, les États africains semblent avoir trouvé, à un moment donné de leur histoire (début des années 2000) la bonne formule dans un contexte de mondialisation, avec la mise en place du NEPAD ${ }^{29}$. Le NEPAD « marque une prise de conscience des États africains dans l'urgence d'une sortie de crise pour le continent africain et il définit la nouvelle orientation qu'ils souhaitent imprimer non seulement à son développement, mais également à la nature des relations qu'ils voudraient tisser avec leurs peuples et les partenaires extérieurs » (Davidson, Christian et Nangnigui, 2006, p. 40).

L'Amérique Latine et son virage politique à gauche s'affirme dans le champ de la communication avec Telesur (une sorte de CNN locale version latino-américaine) lancée en 2005 pour contrer l'influence des médias occidentaux et favoriser l'intégration sous-régionale en Amérique latine. Il ne serait pas superflu de noter que l'avenir de cette chaîne soutenue principalement par le Venezuela (sous Chavez) dépend fortement des convulsions internes de cet État qui n'est pas épargné par les crises sociopolitiques.

Toutes ces velléités ont aussi un fond idéologique : une divergence profonde de vision politique, de valeurs culturelles, qui rendrait actuelle (sans la valider) la grave assertion de Samuel Huntington : le « choc des civilisations » $(1993)^{30}$. Huntington ne partage pas l'optimisme affiché par Fukuyama avec sa thèse de la «fin de l'histoire ». Au contraire, il estime que le monde va vers un «clash» causé par de profondes contradictions aux relents identitaires et religieux. L'auteur décrit plusieurs aires de civilisations distinctes (l'Occident chrétien fait face notamment à l'islamisme, et devra compter avec d'autres cultures distinctes d'origines africaine, hindoue, bouddhiste, chinoise, japonaise et éventuellement latino-américaine) ${ }^{31}$. Selon Huntington, «le réveil religieux intervenu depuis les années 1970, la montée de la mondialisation économique et de la démocratie politique ne vont pas (contrairement aux espoirs naïfs des internationalistes américains), se traduire par un rapprochement des régimes et des peuples. On va vers une nouvelle affirmation de soi, éventuellement agressive, de nouvelles puissances sur le mode du nationalisme identitaire dirigée contre l'Occident jusque-là dominant. Huntington fait également un sort particulier à l'Islam qu'il considère comme en quasi-guerre avec l'Ouest. Pour lui, les frontières de l'islam sont sanglantes et cette aire de civilisation en expansion démographique continuera à provoquer des conflits avec ses voisins jusqu'à l'aboutissement de sa transition démographique $\aleph^{32}$. La conclusion pratique de Samuel Huntington est d'une grande clarté, mais aussi d'un cynisme manifeste : «les pays occidentaux, et donc les ÉtatsUnis et l'Europe qui appartiennent objectivement à la même civilisation, doivent se serrer les coudes afin de faire face ensemble à ces inquiétants adversaires que sont surtout les islamistes issus de la civilisation arabo-musulmane $»^{33}$.

Dans ce contexte d'opposition culturelle évoquée par Huntington, il convient de se demander si des tentatives à l'échelle universelle telle que la Convention sur la diversité culturelle (UNESCO 2005) sont une simple goutte d'eau dans la mer ou une 
réelle avancée de la communication à l'échelle internationale susceptible de favoriser une acceptation réciproque des sociétés humaines et des États dans le monde, dans leurs diversités et leurs différences?

De l'ordre de la guerre froide, on est passé aujourd'hui à un désordre mondial ${ }^{34}$. C'est dans ce désordre mondial qui laisse aux acteurs économiques une totale liberté d'entreprise qu'on vit en 2008 et 2009 la plus grande crise économique et financière depuis 1929. Le pessimisme naissant des Américains est contrebalancé par la fin du règne de Bush et l'arrivée à la Maison Blanche du candidat démocrate Obama dont l'engouement médiatique est à la fois national et international ${ }^{35}$. Il y a eu ici un nouvel espoir qui déborde les frontières des seuls États-Unis. Mais, si le monde espère (prix Nobel de la paix pour le premier président noir des États-Unis, Obama en 2009), les États-Unis eux désespèrent vite de leur nouveau leader. On assiste à une sorte de « dissonance communicationnelle » entre les États-Unis et le reste du monde. Le retour des républicains majoritaires à la chambre des représentants dès 2010 et ensuite au sénat en 2014 en serait un indicateur. Pouvait-on dès lors émettre l'hypothèse d'une peur de "rebasculement» du monde avec le risque de l'arrivée au pouvoir des Républicains après une courte parenthèse Obama? Finalement, l'Amérique finit par rallier une sorte de "nouvelle raison mondiale ", en maintenant le démocrate Obama à la Maison Blanche en novembre 2012. Le monde ne s'en porte pas mieux en termes de paix. Au contraire, les foyers de tensions se sont multipliés, et le terrorisme continue encore de frapper, renforçant une obsession sécuritaire qui n'épargne aucune région du monde aujourd'hui. Dans ce contexte précis, la succession d'Obama ne préoccupe pas seulement les citoyens américains. L'élection de novembre 2016 a opposé la candidate démocrate Hilary Clinton à un candidat républicain atypique, Donald Trump, lequel a promis de remettre en cause la politique étrangère traditionnelle des ÉtatsUnis et de « fermer » le pays aux ressortissants musulmans du monde. La victoire finale de Trump traduira-t-elle un retour vers l'obsession sécuritaire avec une cristallisation accrue des tensions religieuses entretenue par le discours belliqueux à l'endroit des musulmans tenu par le nouveau dirigeant américain?

En définitive, si les États-Unis n'ont aucune peine à favoriser la dynamique globalisante $\mathrm{du}$ capitalisme avec l'expansion de leurs industries et compagnies (surtout celles du secteur des communications, Microsoft, Apple, ITT, CNN) au niveau mondial, au plan politique par contre, ils ne parviennent pas à épauler l'ONU pour assurer la sécurité collective et réaliser une paix définitive. Un climat de suspicion et de méfiance réciproque entre les États-Unis et la Russie de Poutine semble se substituer à la fin de la guerre froide. À ce propos, la crise actuelle en Syrie semble réveiller une vieille nostalgie de volonté de neutralisation et de désir de domination entre superpuissances, mais cette fois-ci avec un avantage diplomatique et militaire certain pour la Russie de Poutine qui se présente comme un acteur incontournable pour la résolution de cette crise devenue un vrai « casse-tête " pour les Nations-Unies. La nouvelle confrontation à distance entre les deux "géants" américain et russe intègre même les nouveaux canaux de communication internationale, si l'on considère les accusations des ÉtatsUnis sur d'éventuelles "cyber-attaques» ou tentatives de piratages de ses systèmes d'informations initiées par la Russie. Des actes qui auraient interféré dans les élections présidentielles américaines de novembre 2016.

50 Le paradoxe de la communication internationale est ainsi résumé: plus on a de techniques de communication, moins on s'entend à l'échelle internationale. Plus il y a 
des échanges favorisés par les technologies de l'information et de la communication, plus les divergences éclatent, produisent de l'incompréhension, de la haine et de la violence. Les deux lectures que l'on se fait de la mondialisation aujourd'hui sont un symbole de ces mésententes que charrie la communication internationale: une contradiction que résume bien Jean-Marie Guéhenno (1999, op. cit., p. 13) qui constate une «incompréhension de plus en plus totale entre ceux qui ont trouvé dans une communauté globale virtuelle une réponse à leur besoin d'identité, et tous ceux, les plus nombreux, qui souffrent de subir les décisions des autres et de ne pas même maitriser leur propre destin ».

51 L'ensemble des questions abordées nous ramène à un constat: une réflexion scientifique sur la communication internationale est inséparable d'une analyse qui la situerait dans l'univers global des sciences sociales, d'où l'on ne peut rigoureusement extraire cette " interdiscipline » ou cette « indiscipline » sans un volontarisme forcené qui confinerait à une sorte de dictature épistémique.

\section{BIBLIOGRAPHIE}

Christian Agbobli, Oumar Kane et Gaby Hsab (dir.), Identités diasporiques et communication, Presses de l'Université du Québec, 2013.

Christian Agbobli et Gaby Hsab (dir.), Communication internationale et communication interculturelle : regards épistémologiques et espaces de pratique, Presses de l'Université du Québec, 2011.

Gado Alzouma, "Téléphone mobile, Internet et développement : l'Afrique dans la société de l'information ?" Tic \& société, vol. 2, n² 2, 2008, pp. 35-58.

Raymond Aron, Les étapes de la pensée sociologique, Gallimard, 1967.

Paul Attallah, Théories de la communication. I, Histoire, contexte, pouvoir, Télé-université, 1989.

Bertrand Badie et Marie-Claude Smouts, Le retournement du monde. Sociologie de la scène internationale, Dalloz-Sirey, 1999.

Mounir Bensalah, Réseaux sociaux et révolutions arabes, Michalon, 2012.

Luc Boltanski et Ève Chiapello, Le nouvel esprit du capitalisme, éd. Gallimard, 1999.

Yannick Bosc, «Liberté et propriété. Sur l'économie politique et le républicanisme de Condorcet », Annales historiques de la Révolution française. En ligne : https://

journals.openedition.org/ahrf/12215, 2011 (page consultée le 24 avril 2016).

Philippe Bouquillion et Jacob Thomas Matthews, Le web collaboratif, mutations des industries de la culture et de la communication, Presses universitaires de Grenoble, 2009. En ligne : http:// questionsdecommunication.revues.org/2875 (page consultée le 24 avril 2016).

Aurélien Boutaud, A, "Les inégalités mondiales : une réalité plurielle", Mouvements, $\mathrm{n}^{\circ} 64$, pp. 13-21, 2010.

Philippe Breton, L'utopie de la communication, La Découverte, 1992. 
Zbigniew Brzezinski, La Révolution technétronique, Calmann-Lévy, Paris, 1970. Pierre Delfaud, Keynes et le keynésianisme, PUF, collection « Que sais-je », 1997.

Orlando Figes, La Révolution russe : 1891-1924 : la tragédie d'un peuple, Denoël, 2007. Francis Fukuyama, La fin de l'histoire et le dernier homme, Paris, Flammarion, 1992.

John Kenneth Galbraith, La crise de 1929 Anatomie d'une catastrophe financière, Payot, 2011.

Cyndy Gallois, Tania Ogay et Giles Howard, « Communication Accommodation Theory : A Look Back and a Look Ahead », dans William Gudykunst (dir.), Theorizing About Intercultural Communication, pp. 121-149, 2005.

Éric George et Fabien Granjon (dir.) Critique de la société de l'information, L'Harmattan, 2008.

William Gudykunst et Bella Mody, Handbook of international and intercultural communication 2, Sage publications, 2002.

Jean-Marie Guéhenno, "Américanisation du monde ou mondialisation de l'Amérique", Politique étrangère, vol. 64, numéro 1, pp. 7-20, 1999.

Jurgen Habermas, De l'éthique de la discussion. Traduit de l'allemand par Mark Hunyadi, Paris, Cerf, collection "Passages", 1992.

Samuel Huntington, "The clash of civilizations", Foreign affairs, vol. 72, n. 3, 1993.

Pierre Lellouche, 1992, Le nouveau monde : de l'ordre de Yalta au désordre des nations, Grasset, 1992.

David Lerner, The Passing of Traditional Society, New York, Free Press, 1968.

Thierry Libaert, La transparence en trompe-l'œil, Paris, éditions Descartes et Cie, 2003. Laurent Licata et Audrey Heine, Introduction à la psychologie interculturelle, de Boeck, 2012.

Ndiaga Loum, « Paradigme de l'interdisciplinarité, communication et développement international », Revue française des sciences de l'information et de la communication [En ligne], $4 \mid$ 2014, URL : http://rfsic.revues.org/961 ; DOI : 10.4000/rfsic.961, 2014.

Ndiaga Loum et Raymond Corriveau, Les rapports de pouvoirs entre le champ médiatique et le champ politique : études de cas. Rapport de recherche effectué dans le cadre du projet « La gouvernance des systèmes de communication », Les Cahiers du CRICIS, 2014-5. Montréal, 2014.

Ndiaga Loum, « Du droit à la communication à la solidarité numérique : questions récurrentes dans des contextes idéologique, politique, économique, technique différents », dans A. Kiyindou et R. Amador (sous la direction), Nouveaux espaces de partage des savoirs. Dynamiques des réseaux et politiques publiques, L'Harmattan, pp. 165-180, 2011.

Ndiaga Loum, «Questionner la solidarité numérique : une tentative de construction théorique » dans C. Agbobli, Quelle communication pour quel changement social, les dessous du changement social, PUQ; pp. 127ᄀ-142, 2010.

Ndiaga Loum, «Les discours sur les NTIC et le développement dans les pays du Sud : le piège récurrent de l'émancipation par le haut ", dans G. Tremblay (sous la direction), L'émancipation hier et aujourd'hui, perspectives françaises et québécoises, Québec, Presses de l'Université du Québec, pp. 213-224, 2009.

Ndiaga Loum, « La participation africaine à la société de l'information : pour une théorie critique », Critiques de la société de l'information, Paris, L'Harmattan, pp. 89-103, 2008.

Sean MC Bride, Voix multiples, un seul monde : communication et société d'aujourd'hui et demain, Paris, Documentation française, UNESCO, 1980. 
Marshal McLuhan, Guerre et paix dans le village global, Laffont, Paris, 1970.

Mustapha Masmoudi, « The New World Information Order », in L. John Martin \& Ray Eldon Hiebert, Current Issues in International Communication, Longman, pp. 311-320, 1990.

Michel Mathien, « L'actualité du nouvel ordre mondial de l'information et de la communication ", Annuaire français de relations internationales, Volume 12, pp. 967-987, 2011.

Armand Mattelart et André Vitalis, Le profilage des populations. Du livret ouvrier au cybercontrôle, La Découverte, 2014.

Armand Mattelart, La mondialisation de la communication, Paris, Presses Universitaires de France, «Que sais-je?», 2008.

Armand Mattelart, La globalisation de la surveillance, aux origines de l'ordre sécuritaire, La Découverte, 2007.

Armand Mattelart, La mondialisation de la communication, PUF, coll. « Que sais-je ? », Paris, 1996.

Armand Mattelart, «Dangereux effets de la globalisation des réseaux », Le Monde Diplomatique, août 1995.

Odile Riondet, Nangnigui David Kamara, Christian Edmon Bepi Pout et Davidson William Taffotien Assanvo, «Les technologies de l'information et de la communication et la diplomatie en Afrique : défis et enjeux », Communication, vol. 28/1 | pp. 308-312, 2010.

Raphaël Ntambue, Internet, son web et son e-mail en Afrique. L'approche critique, L'Harmattan, 2001. Joseph Nye, Bound to lead: The Changing Nature of American Power, New York, Basic books, 1990. Lauraent Palou Lacoste, «La pensée de Paul Ricoeur et le choc des civilisations », (En ligne) : http://www.atlasinfo.fr/La-pensee-de-Paul-Ricoeur-et-le-choc-des-civilisations_a96.html, 2010, (page consultée pour la dernière fois le 10 mai 2016)

Karl Polanyi, La Grande transformation sociale. Aux origines politiques et économiques de notre temps, Gallimard, 1983.

Charlie Post, The American Road to Capitalism, Brill, "Historial Materialism Book Series", 28, Londres et Boston, 2011.

Moishe Postone, Cité dans le temps, travail et domination sociale, éditions Mille et une nuits, Paris, 2009.

Michel Pouget, Taylor et le taylorisme, PUF, 1998.

Serge Proulx et André Vitalis, Vers une citoyenneté simulée : médias, réseaux et mondialisation, Éditions Apogée, Rennes, 1999.

Marc Raboy, « Une régulation démocratique problématique », dans Serge Proulx et André Vitalis (sous la direction de), Vers une citoyenneté simulée, médias, réseaux et mondialisation, éditions Apogée, Rennes, pp57-69, 1999.

Everet Rogers, La Théorie de la diffusion, New-York, Free Press, 1962.

Herbert Schiller, Communication and Cultural Domination, New York, Sharpe, 1976.

Ferdinad Tönnies, Gemeinschaft und Gesellschaft, Leipzig : Fues's Verlag. (Traduit en anglais en 1957 par Charles Price Loomis sous le titre Community and Society, East Lansing : Michigan State University Press), 1887.

André Vitalis, L'incertaine révolution numérique, Iste Éditions, 2016. 
André Vitalis, Informatique, pouvoir et libertés, Economica, 1981.

Immanuel Wallerstein, Impenser la science sociale. Pour sortir du XIXe siècle. Pratiques théoriques (surtout la partie «Questions de paradigmes : un appel à débat », pp. 269-289, 1995.

Immanuel Wallerstein, «L'Occident, le capitalisme et le système-monde moderne ", Sociologie et sociétés, Théorie sociologique de la transition, Volume 22, numéro 1, pp. 15-52, 1990.

Dominique Wolton, L'autre mondialisation, Flammarion, 2003. Dominique Wolton, Penser la communication, Flammarion, 1997.

\section{NOTES}

1. L'analyse fournie sur ce point est Inspirée des distinctions opérées par Tonnies, voir Attallah,

P, Théories de la communication. I, Histoire, contexte, pouvoir, 1989, Télé-université.

2. Tönnies, Ferdinand (1887). Gemeinschaft und Gesellschaft, Leipzig: Fues's Verlag. (Traduit en anglais en 1957 par Charles Price Loomis sous le titre Community and Society, East Lansing: Michigan State University Press).

3. Aron, R. (1967), Les étapes de la pensée sociologique, Gallimard (Durkheim, pp. 362-372).

4. Ibid. voir aussi chapitres consacrés à Pareto (pp. 407-423) et à Weber, (pp. 497-522).

5. Aron, R. (1967), Les étapes de la pensée socio- logique, Gallimard (Montesquieu, pp. 25-53) ; (Comte, pp. 77-105) ; (Marx, pp. 141-157 et pp. 184-202) ; (Tocqueville, pp. 221-229 et pp. 251-262).

6. Ibidem

7. Voir «L'Occident, le capitalisme et le système-monde moderne », Revue Sociologie et sociétés, Théorie sociologique de la transition, 1990, Volume 22, numéro 1, printemps 1990, p. 15-52. Voir aussi Wallerstein, I, 1995, pp. 269-289.

8. Expression découverte pour la première fois dans son ouvrage The medium is the message, publié en 1967, Bantam Books.

9. Selon André Vitalis, le projet politique de la société de l'information « ne doit pas faire illusion. En effet, précise l'auteur, malgré les apparences, ce projet marque en fait une démission du politique devant les logiques techniques et économiques ", dans Proulx, Serge, et André Vitalis, 1999, p. 40.

10. Voir extraits du discours de Truman, dans Éric Berr, «À la recherche d'un autre développement », en ligne: http://gretha.u-bordeaux.fr/sites/default/files/doc_chercheurs/ deq-chap-berr.pdf (consultée le 5 avril 2016).

11. Voir Actes du colloque tenu à Bercy les 21, 22, 23, mars 1991, Le Plan Marshall et le Relèvement économique de l'Europe, Colloque, édité par le Comité pour l'histoire économique et financière de la France, 1993.

12. Voir sur cette question un certain nombre de publications, dont : Loum, N, 2011 ; Voir aussi Masmoudi, 1990, pp. 311-320 ; Mathien, M, 2011 ; voir surtout, MC Bride, Sean, 1980.

13. La situation s'est empirée aujourd'hui, et l'on parle de plus en plus d'une richesse mondiale concentrée entre les mains de « $1 \%$ de la population mondiale». Voir rapport 2015 de l'organisation non gouvernementale Oxfam, «Insatiable richesse, toujours plus pour ceux qui ont déjà tout ». En ligne : https://www.oxfam.org/sites/www.oxfam.org/files/file_attachments/ibwealth-having-all-wan-ting-more-190115-fr.pdf (consultée pour la dernière fois le 5 avril 2016).

14. Voir aussi Marshall McLuhan, Guerre et paix dans le village global, Laffont, Paris, 1970. (en collaboration avec Quentin Fiore) et Zbigniew Brzezinski, La Révolution technétronique, CalmannLévy, Paris, 1970. Pour Breton, la communication en tant que discipline va naître avec les travaux de Wiener vers 1942. Voir Breton, P, 1992, L’utopie de la communication, La Découverte. 
15. Armand Mattelart, 1995, Le Monde Diplomatique, «Dangereux effets de la globalisation des réseaux ", août 1995.

16. Voir notamment Joseph Nye, Bound to lead, 1990 et Francis Fukuyama, La fin du monde, 1992.

17. J e a n-Ma $\mathrm{r}$ ie $\mathrm{G}$ ué $\mathrm{h}$ enn o , 1999, "Américanisation du monde ou mondialisa- tion de l'Amérique ", Politique étrangère, vol. 64, numéro 1, pp. 7-20.

18. Cité par Guehenno, J.M., op.cit, p. 7

19. Bosc, Yannick, 2011, 'Liberté et propriété. Sur l'économie politique et le républicanisme de Condorcet, Annales historiques de la Révolution française", en ligne: https:// journals.openedition.org/ahrf/12215

20. P. Bouquillion et J.T. Matthews, 2009, Le web collaboratif, mutations des industries de la culture et de la communication, Presses universitaires de Grenobl.

21. Herbert Schiller, 1976, Communication et domination culturelle, p. 9.

22. De très nombreux travaux sont faits dans ce domaine précis. Voir notamment William $B$. Gudykunst, W-B, et B, Mody, Handbook of international and intercultural com- munication 2, Sage publications, 2002 ; E. M Rogers et W-B. Hart, « The Histories of Intercultural, International, and Development Communication. », dans Gudykunst \& Mody (Ed.), Handbook of international and intercul-tural communication 2, 2002 ; Licata, L et A, Heine, Introduction à la psychologie interculturelle, de Boeck, 2012 ; C. Gallois, T Ogay et H. Giles, « Communication Accommodation Theory : A Look Back and a Look Ahead»,dans W.B. Gudykunst (dir.), Theorizing About Intercultural Communication, 2005, p. 121-149. Voir aussi Agbobli, C, O. Kane et G. Hsab (sous la direction de), Identités diasporiques et communication, Presses de l'Université du Québec, 2013 et Agbobli, C et G, Hsab (sous la direction de), Communication internationale et communication interculturelle: regards épistémologiques et espaces de pratique, Les Presses de l'Université du Québec, 2011.

23. Voir Loum, N. « Développement et Culture à l'ère du "tout numérique" : une problématique à repenser ou à renouveler?» (Publication à paraître dans Michèle Rioux (sous la direction de), Cultures, Société et Numérique, 2016.

24. Voir la loi dite Patriot Act. Loi antiterroriste votée par le Congrès des États-Unis et signée par George W. Bush le 26 octobre 2001. Voir notamment la section 215 du Patriot Act qui permet aux autorités américaines d'obtenir de la part d'un tribunal secret un mandat qui oblige les opérateurs de services de télécommunications de leur fournir l'intégralité des données téléphoniques sur leurs clients. Une nouvelle loi dite Freedom Act votée en juin 2015 et signée par Barack Obama remplace le Patriot Act en assouplissant les dispositions liées aux écoutes téléphoniques à grande échelle qui violeraient des libertés individuelles sur la seule base de la lutte contre le terrorisme

25. Voir notamment, Vitalis, A, 1981, Informatique, pouvoir et libertés, Economica ; du même auteur, 2016, L'incertaine révolution numérique, Iste Éditions ; Voir également Mattelart, A, 2007 ; Mattelart, A et A. Vitalis, 2014.

26. Les révolutions arabes, notamment celle tunisienne et celle égyptienne auraient révélé un très fort usage des médias sociaux, particu- lièrement Facebook, par les jeunes « révolutionnaires ». Cet usage est aujourd'hui largement documenté, tantôt dans un élan d'optimisme quant au potentiel de ces nouveaux médias, tantôt avec plus de prudence. Voir notamment : Bensalah, M, 2012, Réseaux sociaux et révolutions arabes, Michalon. L'auteur estime dans cet ouvrage que le rôle des médias sociaux durant le Printemps arabe a été très exagéré, notamment par les médias occidentaux.

27. Voir Daniel Lerner, dans son ouvrage, The Passing of Traditional Society, publié en 1958, New York, Free Press. Sa théorie, dite paradigme de la modernisation, soutient que seul un transfert de technologies des pays industrialisés vers les pays pauvres est capable d'amener les pays du tiers-monde à réduire leur retard et de réaliser le développement. En 1962, Everett Rogers, dans un ouvrage intitulé La Théorie de la diffusion, New-York, Free Press, va abonder dans le même sens que Lerner. Rogers considère la modernisation comme un processus de diffusion des techniques 
permettant aux populations de passer d'un mode de vie différent, plus développé sur le plan technologique et plus adapté aux changements rapides.

28. Voir Boutaud, Aurélien, 2010 ; Loum, N. « Questionner la solidarité numérique : une tentative de construction théorique" dans C. Agbobli, 2010 ; Loum, N, 2014 ; 2009; 2008. Voir aussi R. Ntambue, 2001 ; G. Alzouma, », 2008 ; A. Chéneau-Loquay, 2007.

29. Nouveaupartenariat pour le développement de l'Afrique initié par les anciens présidents sénégalais, Wade, nigérian, Obasanjo, sud-africain, Mbeki et le président algérien Bouteflika.

30. Huntington, S., "The clash of civili- zations", Foreign affairs, 1993, vol. 72, n. 3, pp. 22-49.

31. Voir Laurent Palou Lacoste, "La pensée de Paul Ricoeur et le choc des civilisations ", en ligne : http://www.atlasinfo.fr/La-pensee-de-Paul-Ricoeur-et-le-choc-des-civilisations_a96.html.

32. Ibidem.

33. Ibidem.

34. Voir Pierre Lellouche, 1992 ; Bertrand Badie et Marie-Claude Smouts parlent à leur tour d'un « monde privé de sens » et de « retournement du monde » (Voir Badie, B et Smouts, M.C., 1999).

35. Voir cérémonie d'investiture du premier président noir des États-Unis, Obama, retransmise dans 160 pays; une couverture médiatique en $24 \mathrm{~h}$ qui serait équivalente à 3 ans de contenu médiatique dans la presse au Québec.

\section{RÉSUMÉS}

Communication internationale ou internationalisation de la communication? À quelque bout qu'on prend la question, la pertinence de l'existence de la communication internationale comme sous-discipline, discipline ou matière se pose dans l'univers global des sciences sociales. Si l'on reconnaît une discipline scientifique à travers les diplômes qui sanctionnent un cursus académique prédéfini, par l'ampleur des travaux qui portent le sceau de la matière désignée, par le nombre de chercheurs revendiquant une telle spécialité ou reconnus comme tels, par le nombre de centres de recherche abritant lesdits spécialistes, il est démontré que la pertinence de l'enseignement et de la recherche en communication internationale ne fait pas l'objet d'un doute. Ceci explique d'ailleurs que ce débat, s'il a existé un moment, n'est plus un centre d'intérêt réel. Ce qui l'est par contre, c'est le carrefour interdisciplinaire que constitue cette sous-discipline «ballotée» entre sa source de rattachement ombilical (la communication), les relations internationales qu'elle "chevauche " sans risque d'entremêlement, et la science sociale qui englobe l'ensemble des recherches touchant la société lato sensu et qu'elle ne peut ignorer. Comment la communication internationale peut revendiquer une place de choix dans l'univers global des sciences sociales? Dans quelle mesure et jusqu'à quel point les objets qu'elle aborde, les enjeux sociétaux qu'elle adresse interpellent-ils toutes les disciplines des sciences sociales? Comment démontrer sans effort son rattachement à la trajectoire historique que suivent les sciences sociales? C'est dans cette disposition d'esprit que s'enclenche cette réflexion quasi heuristique qui porte sur des questions d'épistémologie autour d'une "indiscipline » ou d'une « interdiscipline » nommée communication internationale.

International communication or internationalization of communication? At some point, the relevance of the existence of international communication as a sub-discipline, discipline or subject matter arises in the global universe of the social sciences. If a scientific discipline is recognized through the diplomas awarded by a predefined academic curriculum, by the extent of 
the work that bears the seal of the subject, by the number of researchers claiming such a specialty or recognized as such, Number of research centers in which these specialists are located, it is demonstrated that there is no doubt as to the relevance of teaching and research in international communication. This explains why this debate, if it existed for a moment, is no longer a real center of interest. What is the interdisciplinary crossroads of this sub-discipline "tumbled" between its source of umbilical attachment (communication), the international relations it "overlaps" without risk of entanglement, and The social science which encompasses all research concerning society lato sensu and which it can not ignore. How can international communication claim a prominent place in the global universe of the social sciences? To what extent and to what extent are the social issues addressed by it addressed by all the disciplines of the social sciences? How can one demonstrate without effort its attachment to the historical trajectory followed by the social sciences? It is in this perspective that this quasi-heuristic analysis begins which concerns epistemological questions around an "indiscipline" or an "interdiscipline" called international communication.

Comunicação internacional ou comunicação internacional? Em qualquer maneira que você toma a questão, a relevância da existência de comunicação internacional como uma sub-disciplina, disciplina ou assunto surge no universo total de ciências sociais. Se uma disciplina científica é reconhecida pelo diplomas premiado com um curso pré-acadêmica, pela quantidade de trabalho que carregam o selo do material designado, o número de pesquisadores que afirmam tais especialidade ou reconhecido como tal pela número de centros de pesquisa que abrigam os chamados peritos, há evidências de que a relevância do ensino e da pesquisa em comunicação internacional não está sujeita à dúvida. Isto explica também que este debate, se existisse algum tempo, não é, mais um centro de interesse real. o que é os contras é a encruzilhada interdisciplinares que constituem este sub-disciplina "jogou" entre a sua fonte de ligação umbilical (comunicação), relações internacionais, ela "passeios" sem risco de enrolamento e ciência social que engloba todas as pesquisas envolvendo a sociedade em sentido amplo e que não podemos ignorar. Como a comunicação internacional pode reivindicar um lugar no universo total de ciências sociais? Como e em que medida os objetos que abrange, tratar de questões sociais que desafiam todas as disciplinas das ciências sociais? Como facilmente demonstrar o seu apego à trajetória histórica que siga as ciências sociais? É neste estado de espírito que se encaixa esta reflexão heurística quase que aborda questões de epistemologia em torno de uma "indiscipline" ou "interdiscipline" nomeado comunicação internacional.

INDEX

Mots-clés : communication internationale, mondialisation, sciences sociales, histoire, développement, capitalisme

Keywords : international communication, globalization, social sciences, history, development, capitalism

Palavras-chave : comunicação internacional, globalização, ciências sociais, história, desenvolvimento, capitalismo

\section{AUTEUR}

NDIAGA LOUM

Université du Québec en Outaouais 